\title{
A new method for critical path method with fuzzy processing time
}

\author{
N. Shahsavari Pour ${ }^{\mathrm{a}}$, M. Kheranmand ${ }^{\mathrm{b} *}$, M. Fallah ${ }^{\mathrm{c}}$ and S. Zeynali ${ }^{\mathrm{d}}$
}

${ }^{a}$ Department of Industrial Engineering, Islamic Azad University - Kerman Branch, Kerman, Iran

${ }^{b}$ Department of Industrial Engineering, Islamic Azad University - Science and Research Branch, Tehran, Iran

${ }^{c}$ Department of Industrial Engineering, Islamic Azad University - South Tehran Branch, Tehran, Iran

${ }^{d}$ Department of Industrial Engineering, Islamic Azad University - Science and Research Branch, Tehran, Iran

A R T I C L E I N O A S T R A T

Article history:

Received October 292010

Received in revised form

15 January 2011

Accepted 10 February 2011

Available online

15 February 2011

Keywords:

Critical path method

Fuzzy numbers

Fuzzy theory

Uncertainty

\begin{abstract}
Critical path method plays an important role on managing medium to large-scale problems. It is often difficult to determine the critical path for different reasons such as the existing uncertainties in processing tasks. One alternative to handle the uncertainty associated with the processing time is to use fuzzy techniques. We present a new method to calculate the critical path method when the processing times follow trapezoidal fuzzy numbers. The proposed model of this paper does not use any defuzzification technique to find the final processing time. The implementation of the proposed model is compared with other techniques using a well-known example from the literature.
\end{abstract}

(C) 2011 Growing Science Ltd. All rights reserved.

\section{Introduction}

During the past few decades, there have been tremendous efforts for providing sophisticated techniques to manage scheduling of projects. In fact, project scheduling is one of the key items for the success of a project. Critical path method (CPM) is an essential tool to determine the total time needed to complete a project (Dean, 1985). CPM splits a project into different activities where each one needs a certain amount of time to complete and determines the critical path based on backward and forward computations. However, the results of a simple implementation of CPM may change since the processing time for each activity could be subject to uncertainty. In other word, when the processing time of a particular activity changes, the critical path may change as well. There are different techniques to handle uncertainty in CPM problem such as program evaluation review technique (PERT) and graphical evaluation review technique (GERT) (Kerzner, 2003). In the first method, PERT, the processing times associated with different activities normally follow a probability

* Corresponding author. Tel: +989124252899

E-mail addresses: Mansour Kheradmand@yahoo.com (M. Kheradmand) 
distribution while in the second method, GERT, the outcome of an activity is uncertain. There are literally various methods similar to PERT method when the processing times are studied with uncertainties. Bertsimas and Sim (2003) developed a robust technique to handle the uncertainty and examined their method on CPM problems. Ben-Tal and Nemirovski (2000) used an interior based method to handle the uncertainty for linear programming problems. These method have become popular approaches and there are various applied works associated with them (Sadjadi \& Omrani, 2008; Gharakhani et al., 2010). The other method to handle the uncertainty is to use fuzzy programming (Zadeh, 1965). Fuzzy technique is more suitable when we do not have a previous experience on the input data and the data is derived based on expert's insight. Chanas and Zieliński (2001) presented two techniques for the calculations of the path degree of criticality. Dubois et al. (2003) presented fuzzy intervals model with uncertain durations of tasks for CPM problem based on possibility theory. Zieliński (2005) proposed a method to compute the intervals of possible values of the latest starting times and floats of activities in the networks with imprecise durations, which are expressed by either interval or fuzzy numbers. The method is polynomial and it is more suitable to determine the latest starting times for a general network. Lin and Yao (2003) presented a fuzzy CPM based on signed-distance ranking and statistical confidence-interval estimates. Shahsavari pour et al. (2010) studied fuzzy CPM problem and presented a method where the approach does not need any defuzzification technique in any part of the implementation of their algorithm. This paper presents another fuzzy CPM where the processing times of all activities follow trapezoidal fuzzy numbers.

The organization of this paper first presents problem statement in section 2. Section 3 explains the proposed algorithm and the implementation of the proposed model is illustrated using a numerical example. Finally, concluding remarks are given in the last section to summarize the contribution of the paper.

\section{Problem statement}

Consider a network with different tasks where each task has a processing time, which follows fuzzy value. The proposed model of this paper uses trapezoid fuzzy number $\tilde{A}$ with the following membership function for each activity,

$$
\mu_{\widetilde{A}}(x)=\left\{\begin{array}{cc}
1-\left(a_{1}-x\right) / \alpha & a_{1}-\alpha \leq x \leq a_{1} \\
1 & x \in\left[a_{1}, a_{2}\right] \\
1-\left(x-a_{2}\right) / \beta & a_{2} \leq x \leq a_{2}+\beta
\end{array}\right.
$$

Fig 1. Shows the details of the trapezoid number with $\tilde{A}=\left(a_{1}, a_{2}, \alpha, \beta\right)$.

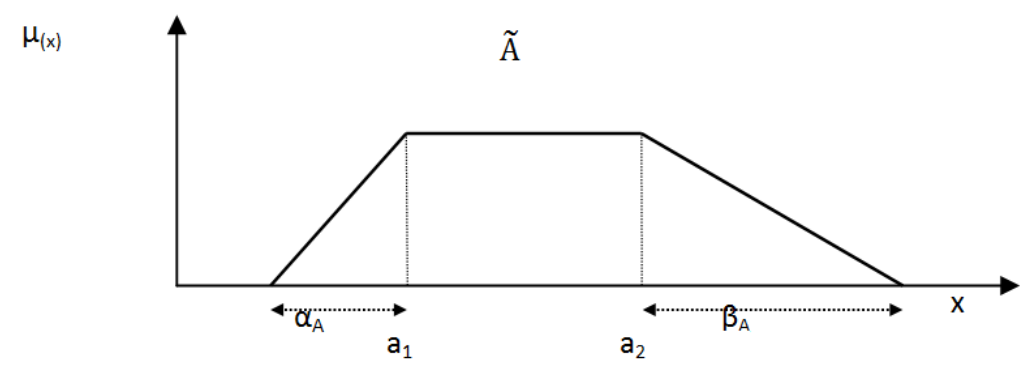

Fig. 1. Trapezoid number $\tilde{A}$ 
According to Zadeh (1965), two trapezoid fuzzy numbers $\tilde{A}=\left(a_{1}, a_{2}, \alpha_{A}, \beta_{A}\right)$ and $\tilde{B}=\left(b_{1}, b_{2}, \alpha_{B}, \beta_{B}\right)$ are added as $\tilde{A}+\tilde{B}=\left(a_{1}+b_{1}, a_{2}+b_{2}, \alpha_{A}+\alpha_{B}, \beta_{A}+\beta_{B}\right)$. Let $x_{1 A}$ and $x_{2 A}$ be two fuzzy numbers which are associated with $\tilde{A}$ and $x_{1 B}$ and $x_{2 B}$ be two fuzzy numbers which are associated with $\widetilde{B}$. In order to compare two numbers we first calculate the following for addition of two numbers,

$$
\begin{aligned}
& \left.\begin{array}{l}
x_{2 A}=a_{2}+(1-y) \beta_{A} \\
x_{1 A}=a_{1}+(y-1) \alpha_{A}
\end{array}\right\} \text { for } \widetilde{\mathrm{A}} \\
& \left.\begin{array}{rl}
x_{2 B} & =b_{2}+(1-y) \beta_{B} \\
x_{1 B} & =b_{1}+(y-1) \alpha_{B}
\end{array}\right\} \text { for } \widetilde{\mathrm{B}}
\end{aligned}
$$

where $y \in(0,1)$. In order to compare two fuzzy numbers we add an arbitrary number $\lambda \in(0,1)$ to $y$ and smaller values are desirable values. Fig. 2 shows two fuzzy numbers.

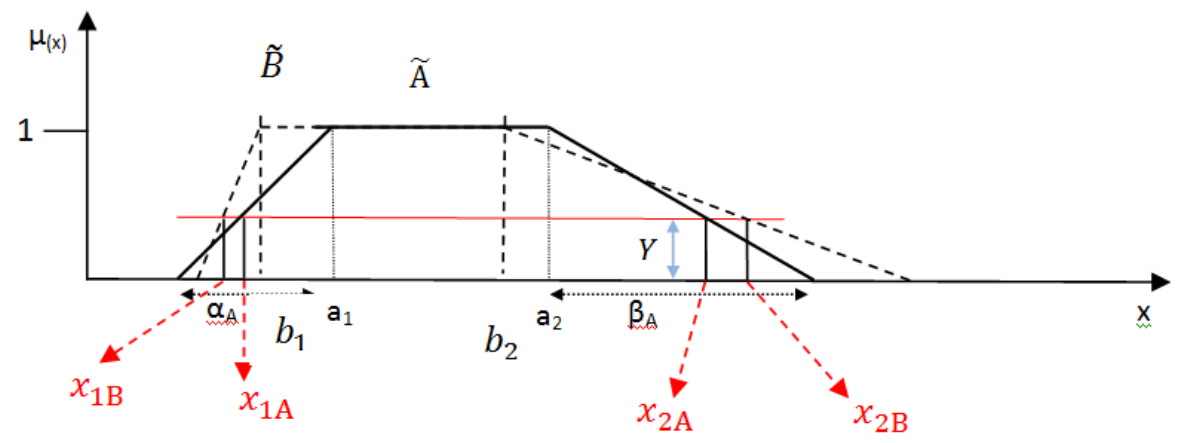

Fig. 2. Two fuzzy numbers

In order to compare two trapezoid numbers we first compare $x_{2 A}$ and $x_{2 B}$ computed by Eq. (3) for a given $y$ and we increase $y$ by $y+\lambda$ and the process is repeated several times while we consider bigger numbers and choose the one with higher number of bigger numbers. In case we do not reach to a conclusion we use $x_{1 \mathrm{~A}}$ and $x_{1 \mathrm{~B}}$. The details of the comparison between fuzzy numbers are given in fig 3.

\section{Proposed method}

Let $n$ be the number of nodes and $A_{n \times n}=\left[a_{i j}\right]$ be the connection matrix with the following notations,

$a_{i j}=\left\{\begin{array}{cc}1 & \text { if node } i \text { starts arc } j \\ 0 & \text { otherwise }\end{array}\right.$

According to Eq. (4), when there is a connection between node $i$ and node $j$ then we have $a_{i j}=1$. Once all processing times are calculated based on the trapezoid numbers and the necessary calculations are performed using the method explained in Fig. 3 we calculate the path using the 
procedure explained in Fig 4. The implementation of the proposed model is shown in the next section.

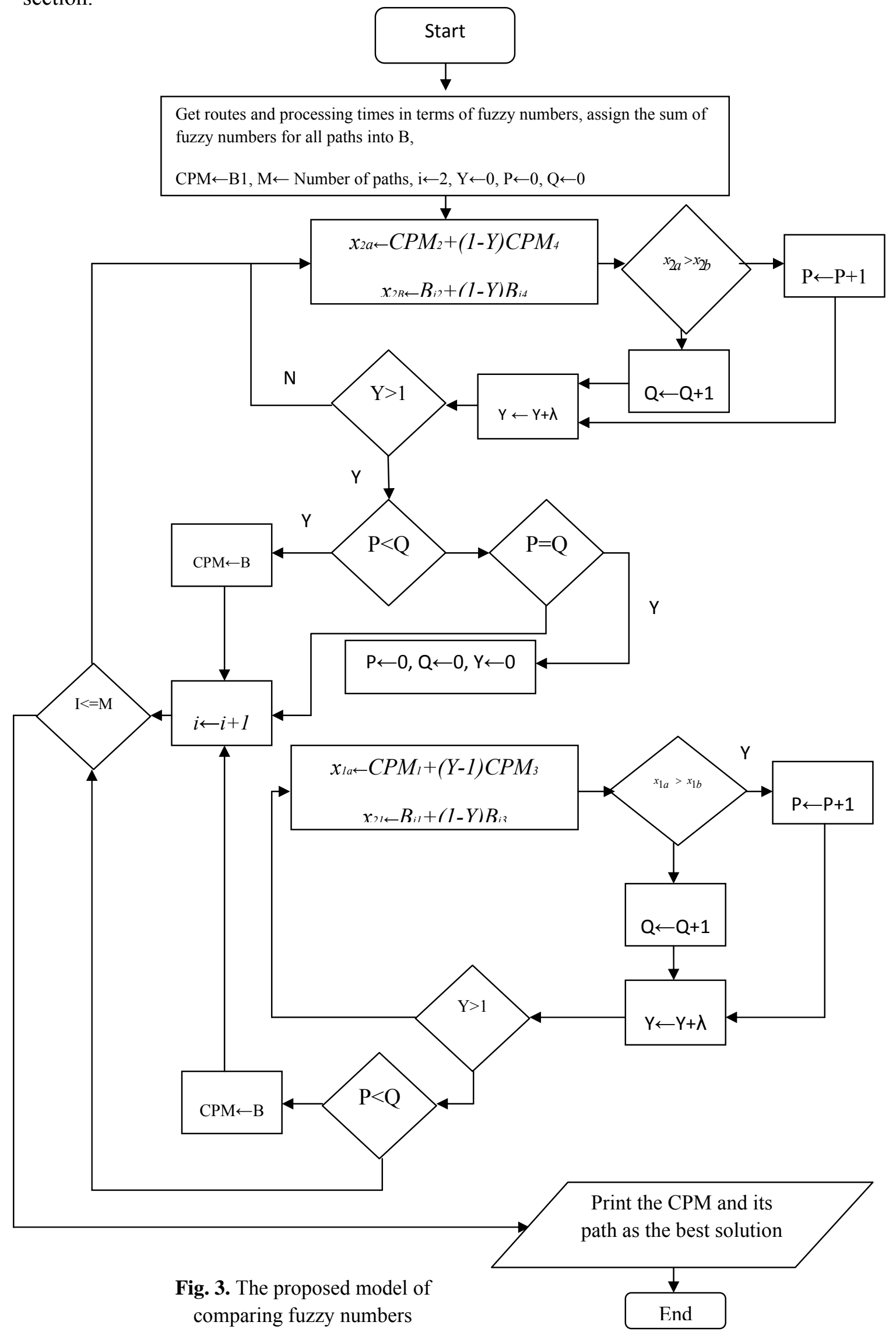




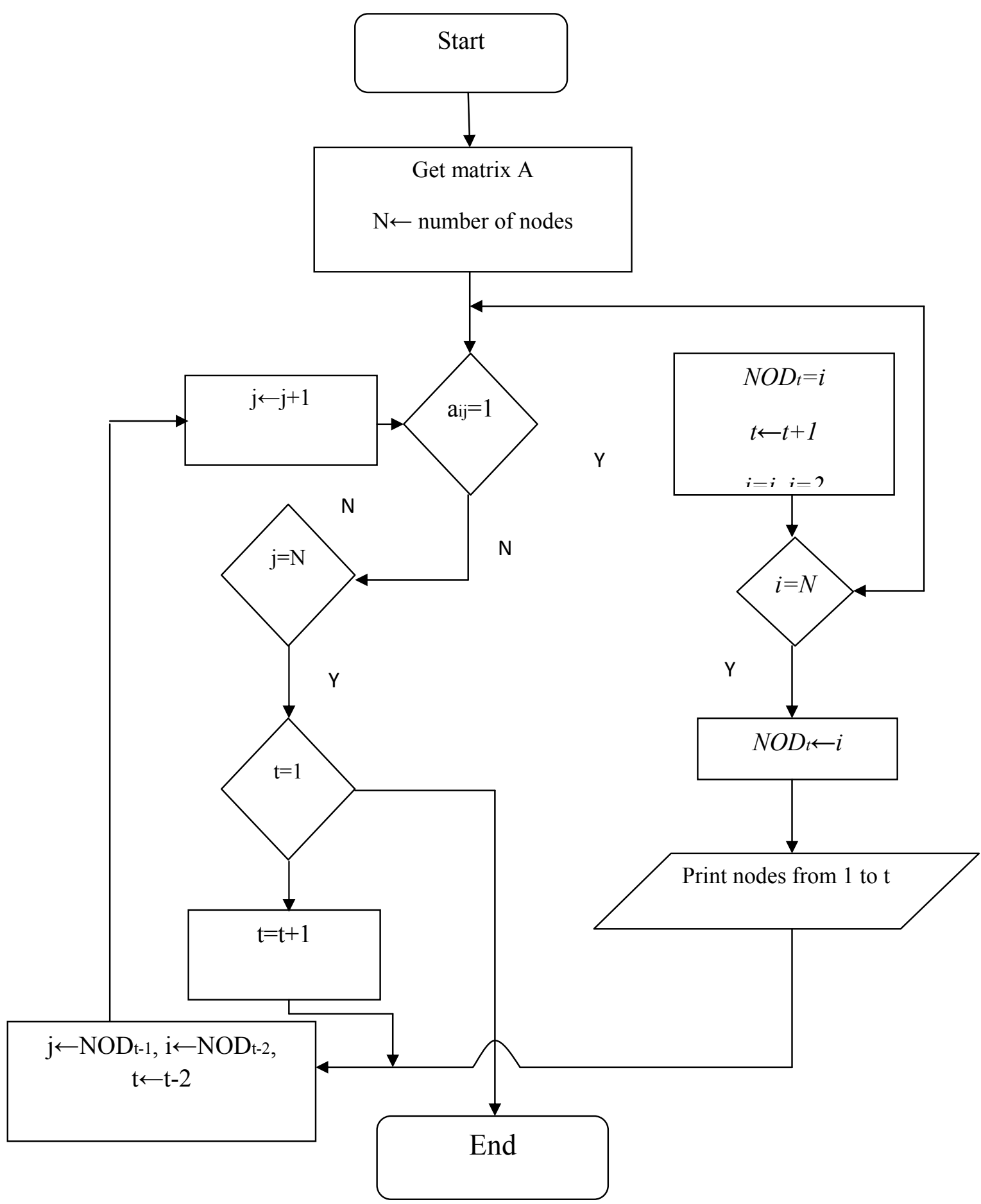

Fig. 4. Determination of paths

\section{Numerical solution}

In this section, we use a well known example in the literature which was already considered by different people (Chanas \& Zieliński, 2001; Chen \& Hsueh, 2008; Shahsavari pour et al., 2010). Consider a network given in Fig 5. 


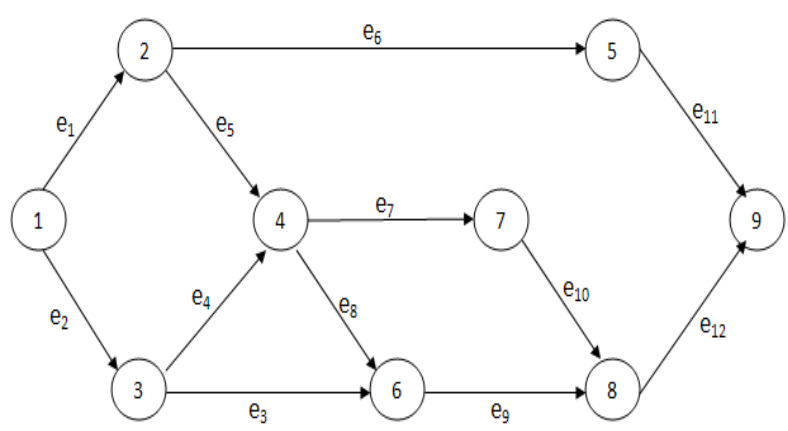

Fig. 5. The network flow of the numerical example

The processing times associated with nodes 1 to 12 are as follows,

$$
\begin{aligned}
& \tilde{\mathrm{t}}_{1}=(1,1.5,1,1), \tilde{\mathrm{t}}_{2}=(2,3,0,2), \tilde{\mathrm{t}}_{3}=(6,7,0,2), \tilde{\mathrm{t}}_{4}=(0,0,0,0) \\
& \tilde{\mathrm{t}}_{5}=(0,0,0,0), \tilde{\mathrm{t}}_{6}=(2,3,1,2), \tilde{\mathrm{t}}_{7}=(9,9,1,1), \tilde{\mathrm{t}}_{8}=(5,5,1,1) \\
& \tilde{\mathrm{t}}_{9}=(4,4,2,2), \tilde{\mathrm{t}}_{10}=(3,4,2,0), \tilde{\mathrm{t}}_{11}=(8,9,2,4), \tilde{\mathrm{t}}_{12}=(6,9,2,3) .
\end{aligned}
$$

The network can be represented in the form of matrix notation as follows,

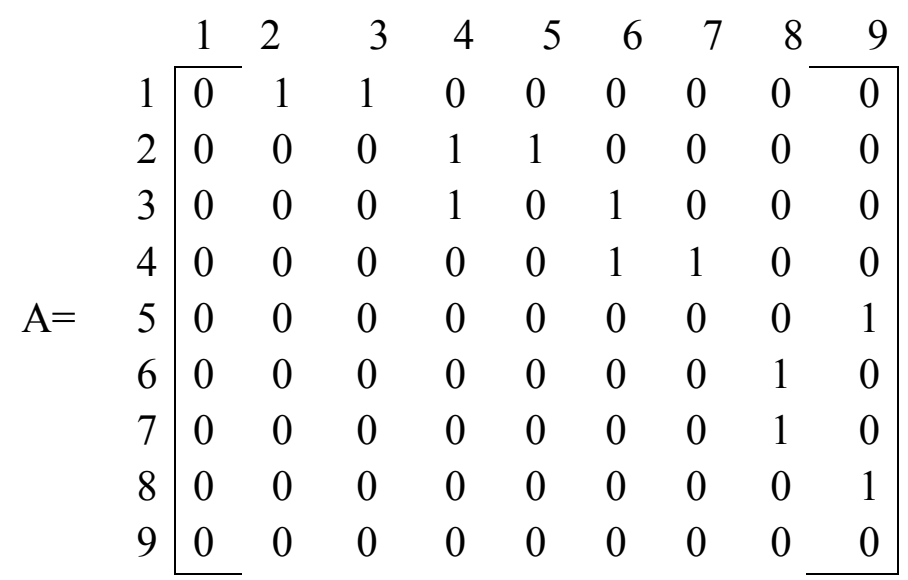

Table 1 summarizes the results of the implementation of the methods proposed by Chanas and Zieliński (2001) and Chen and Hsueh (2008) for the example of this paper.

Table 1

The summary of the network solution

\begin{tabular}{cccc}
\hline \multicolumn{2}{c}{ Chanas \& Zieliński } & Chen \& Hsueh & \\
path & $\mu_{\tilde{p}}(p)$ & path & $\mu_{\tilde{p}}(p)$ \\
\hline $1-2-5-9$ & 0.6269 & $1-3-4-7-8-9$ & 1 \\
$1-2-4-7-8-9$ & 0.5001 & $1-3-6-8-9$ & 0.9574 \\
$1-2-4-6-8-9$ & 0.3854 & $1-2-4-7-8-9$ & 0.9212 \\
$1-3-4-7-8-9$ & 1 & $1-3-4-6-8-9$ & 0.8789 \\
$1-3-4-6-8-9$ & 0.0001 & $1-2-4-6-8-9$ & 0.8001 \\
$1-3-6-8-9$ & 0.9941 & $1-2-5-9$ & 0.5717 \\
\hline
\end{tabular}


The first and the thirds columns of Table1 represent the paths based on different memberships given on the second and the fourth columns of Table.

The proposed model of this paper was solved for this example and the total processing time of the example is calculated in a form of trapezoid number as $(20,25,5,4)$ with the critical path of $(1,3,4,7,8,9)$. This answer is precisely the same as the one we get from the results reported on Table 1 when the membership function is one. One advantage of using the proposed method is that there is no defuzzification on any part of our implementation. Therefore, the results would be more practical for many real-world problems.

\section{Conclusion}

We have presented a new method to calculate the critical path method when the processing times follow trapezoidal fuzzy numbers. The proposed model of this paper does not use any defuzzification technique to find the final processing time. The implementation of the proposed model has been compared with other techniques using a well-known example from the literature. The idea of considering processing times with uncertainty can be extended for CPM problems using other techniques such as scenario planning robust optimization techniques and it can be considered as future research.

\section{Acknowledgment}

The authors would like to thank the anonymous referees for their constructive comments on earlier version of this work.

\section{References}

Bertsimas, D., \& Sim, M. (2003). Robust discrete optimization and network flows, Mathematical Programming Series B, 98, 49-71.

Ben-Tal, A., \& Nemirovski, A. (2000). Robust solutions of linear programming problems contaminated with uncertain data. Mathematical Programming, 88, 411- 421.

Chanas, S., \& Zieliński, P. (2001). Critical path analysis in the network with fuzzy activity times. Fuzzy Sets and Systems, 122, 195-204.

Chen, S-P., \& Hsueh, Y-J. (2008). A sample approach to fuzzy critical path analysis in project networks, Applied Mathematical Modeling, 32(7), 1289-1297.

Dean, B.V. (1985). Project Management: Methods and Studies, North-Holland, Amsterdam.

Dubois, D., Fargier, H., \& Galvagnon, V. (2003). On latest starting times and floats in activity networks with ill-known durations. European Journal of Operational Research, 147(2), 266-280.

Gharakhani, M., Taghipour, T., \& Jalali Farahani, K. (2010). A robust multi-objective production planning. International Journal of Industrial Engineering Computations, 1(1), 73-78.

Kerzner, H. (2003). Project Management: A Systems Approach to Planning, Scheduling, and Controlling (8th Ed. ed.). Wiley.

Lin, F-T., \& Yao, J-S. (2003). Fuzzy critical path method based on signed-distance ranking and statistical confidence-interval estimates. The Journal of Supercomputing, 24(3), 305-325.

Modarres, M. \& Sadi-Nezhad, S. (2001). Ranking fuzzy numbers by preference ratio. Fuzzy Sets and Systems, 118(3), 429-436.

Shahsavari pour, N., Modarres, M. \& Tavakkoli-Moghadam, R. (2010). The discrete time-costquality trade-off using a novel hybrid genetic algorithm. Applied Mathematical Sciences, 4(42), 2081-2094. 
Shahsavari pour, N., Modarres, M., Aryanejad, M. B., \& Tavakoli Moghadam, R. (2010). Calculating the project network critical path in uncertainty conditions, International Journal of Engineering and Technology, 2(2), 136-140.

Sadjadi, S.J., \& Omrani, H. (2008). Data envelopment analysis with uncertain data: An application for Iranian electricity distribution companies, Energy Policy, 36, 4247- 4254.

Zadeh, L. A. (1965). Fuzzy sets, Information and Control, 8 (3), 338-353.

Zieliński, P. (2005). On computing the latest starting times and floats of activities in a network with imprecise durations, Fuzzy Sets and Systems, 150, 53-76. 